\title{
"Homo Caloricus": La construcción epistemológica del estigma lipofóbico en los discursos mediáticos de salud pública
}

\author{
"Homo Caloricus": The epistemological construction of \\ lipophobic stigma in public health media discourses
}

'Licenciada en Filosofía, Doctora en Filosofía de la Ciencia. Docente Titular Departamento de Filosofía, Universidad de Guanajuato. México. $\bowtie$ iD
RESUMEN El caso de la obesidad constituye, en México, un ejemplo emblemático sobre cómo prevalecen, en los mensajes emitidos por las campañas televisivas de salud pública, creencias fuertemente arraigadas en torno a la cuestión del peso corporal. El objetivo principal de este trabajo fue reflexionar sobre ciertos efectos epistemológicos que abundan en los relatos mediáticos biomédicos referidos a la obesidad. Para ello, se analizan algunos aspectos de la retórica propulsada por dos iniciativas de la Secretaría de Salud Pública Muévete y Métete en Cintura, de 2008 y el programa 5 Pasos, de 2011- y por el discurso que giró en torno a la medida impositiva instaurada por el Servicio de Atención Tributaria del gobierno mexicano, en 2017. Por otra parte, este estudio se amplía a otras prácticas similares que, aunque teniendo lugar fuera de México, contribuyen a aceitar los engranajes del lipofobismo, como la "industria Cormillot" en Argentina. Como resultado, se ha identificado un fuerte influjo discursivo emanado de una visión reduccionista sobre la diversidad corporal, sostenido por la patologización de los cuerpos gordos mediante prejuicios morales y generalizaciones sesgadas.

PALABRAS CLAVES Peso Corporal; Índice de Masa Corporal; Medios de Comunicación; Programas de Gobierno; México.

\begin{abstract}
The case of obesity in Mexico constitutes an emblematic example of how, in the messages emitted by public health television campaigns, strong beliefs regarding the issue of body weight prevail. The main objective is to reflect on certain epistemological effects that abound in biomedical media narratives related to obesity. To do so, aspects of the rhetoric employed in two initiatives of the Ministry of Public Health - Muévete y Métete en Cintura [Move Around and Slim Down] in 2008 and the 5 Steps Program in 2011 - are analyzed, as is the discourse surrounding the tax measure introduced by the Mexican tax service in 2017. This study also looks at other similar practices that, although taking place outside of Mexico, stoke the fires of lipophobism, such as the "Cormillot industry" in Argentina. As a result, a strong discursive influx stemming from a reductionist vision of bodily diversity has been identified, supported by the pathologization of fat bodies through moral prejudices and biased generalizations.
\end{abstract}

KEY WORDS Body Weight; Body Mass Index; Communications Media; Government Programs; Mexico. 


\section{INTRODUCCIÓN}

Mucho se ha escrito acerca del inagotable poder del lenguaje para influir e influirnos. Las palabras no cumplen únicamente con la expectativa de "representar" hechos o cosas, sino que pretenden, fundamentalmente, generar efectos. A través del uso específico de las palabras en contextos determinados, pasamos de la supuesta descripción objetiva e imparcial a la evaluación y enjuiciamiento de ciertos cursos de acción (incluso solapada o inadvertidamente). En tanto participantes, usuarios y creadores de la intrincada red de significados dentro de la cual transcurren nuestras vivencias, generamos marcos categoriales, sistemas clasificatorios y patrones explicativos que ponderamos como funcionales para nuestra necesidad de entender, controlar y predecir el temible terreno de lo incierto. El lenguaje posibilita los logros humanos más acabados, pero también las miserias más mundanas:

La misma facultad que permite arreglar una fecha, nos hace difícil dejar de imaginar distintas historias preocupantes si la otra persona Ilega tarde. Un oso puede quedar atrapado en una trampa, pero, al no poseer el "don" del lenguaje, no se pregunta por qué se levantó esa mañana. [...] El lenguaje hace que la gente se abra a formas más penetrantes de sufrimiento y desesperación que los miembros de otras especies. ${ }^{(1)}$

Como ya lo reconocieron algunos filósofos desde antaño, no son los "hechos" los que nos hieren, sino nuestra particular forma de interpretarlos. Por ello mismo, hay que considerar que el lenguaje puede cambiarlo todo; citando a Humberto Maturana:

...el lenguaje duele: golpear a una persona con un trozo de lenguaje puede ser tan potente como hacerlo con un pedazo de madera [...]. Las palabras, al igual que las balas de un arma, cambian la estructura de las personas, y sus vidas". ${ }^{(1)}$
Sin duda, esa capacidad pragmática que el lenguaje encapsula para crear ideas y sembrar "obviedades", no opera en un vacío social; por el contrario, el influjo de las palabras depende casi directamente del tipo de relación que el nombrado mantiene con quien lo nombra. Si este último no personifica, por alguna razón, una autoridad reconocida como tal, su enunciación no nos generaría ninguna credibilidad y, por lo tanto, tampoco nos despertaría preocupación alguna. Las palabras, adjetivos o etiquetas ejercen un efecto cuestionador, inspirador o transformador cuando emanan de una figura en la cual se deposita una indispensable confianza en sus creencias.

Hoy por hoy, la credibilidad más indiscutible proviene del conocimiento científico. A quien esgrime un juicio basado en alguna experticia profesional sobre el dominio científico de un tema se le adjudica un rol privilegiado. Por ello, la descripción y clasificación que un profesional podría ofrecer acerca de nuestra conducta, nuestro estilo de vida, nuestras decisiones, o nuestros pensamientos, tienden a impactar con tanta fuerza sobre nuestra específica y moldeable forma de percibirnos. Ser "profesionalmente" situado dentro de la normalidad o fuera de ella puede significar la diferencia entre aceptarse o despreciarse a sí mismo.

lan Hacking ${ }^{(2)}$ enumeró una serie de operaciones que funcionan de forma articulada para producir un estado de cosas tan idolátrico como este. Con frecuencia, somos proclives a conferirle al conocimiento científico un elevadísimo grado de pureza, así como a dar por sentado que sus grandes logros son resultado de investigaciones metódicas cuyo éxito se explica racionalmente en virtud del cumplimiento de reglas epistémicas estrictamente suscriptas. Pues bien, muchas de esas estrategias epistémicas (que, desde la percepción pública de la ciencia, actúan como admirables "motores de descubrimientos") funcionan, al mismo tiempo, como "modeladores de subjetividades". En otras palabras, generan formas de pensar que se convierten en auténticos oasis clasificatorios, ejerciendo una abrumadora influencia sobre aquellos 
a quienes clasifica. En muchos contextos profesionales, el "sentido del yo" no sale indemne del diagnóstico bajo el cual queda encerrado el individuo. Muy probablemente, tras la "descripción objetiva" del especialista, la subjetividad no será la misma (como tampoco lo será el trato recibido de los demás).

Los engranajes epistemológicos que le inyectan fuerza a la gran maquinaria del conocimiento son diversos y cambiantes. Pero, si algo coadyuva a engrosar la legitimidad de la voz científica, es la desmedida complacencia que se le otorga a la formación académica del especialista, acreditada por una red de instituciones que fungen como garantes de tal solvencia y en las cuales tendemos a confiar sin más. Como bien explica Hacking( ${ }^{(2)}$, los conocimientos que el profesional detenta se presentan como respaldados en complejos procesos explicativos (a través de la formulación de teorías causales), abrevando del conteo, la cuantificación y la correlación (entre otras operaciones útiles al aumento de la matematización del fenómeno estudiado). Y, cuando lo que se trata de entender es la condición humana y la conducta social, se privilegiará frecuentemente el recurso epistémico que mejor pareciera cumplir con las prerrogativas de un conocimiento que esté a la altura de las exigencias de la objetividad. Por ello mismo, las explicaciones biológicas, médicas o genéticas, han ido ganando una preeminencia profesional que, al mismo tiempo, allana el camino para la administración burocrática del problema en cuestión ${ }^{(2,3,4,5,5,6,7,8)}$.

No son pocas las disciplinas que, abocadas a la comprensión de la condición de la salud, han buscado avanzar por esa senda encaminada a la estandarización. La infraestructura estadística ha sido, en tales casos, la clave para la consolidación de muchos campos de estudio que se han visto empoderados a través de sus estrechas ligazones con la medicina, la cual ha instalado en el sentido común la idea de que "lo sano" y lo "normal" (en el sentido de promedio estadístico) son equiparables. Vista de cerca, tal equiparación conlleva implicaciones sumamente cuestionables. Por ejemplo, si lo estadísticamente típico es por ello saludable, también deberíamos aceptar que la desviación de una conducta con respecto a esa norma estadística es no-saludable (patológica).

Sin embargo, como ya lo advirtió George Canguilhem en su magistral obra Lo Normal y lo patológico ${ }^{(9)}$ existen anomalías (desviaciones del promedio típico) que no merman la calidad de vida del individuo que la posee, ni le imponen límites o restricciones y, por lo tanto, no podrían ser tratadas como patologías. Podría incluso suceder que esas anomalías constituyan, para ese individuo, auténticos "logros" que aumentan sus posibilidades de elegir cómo vivir (las rarezas no son intrínsecamente patológicas, ya que pueden ser incluso creativas). Como esgrime Canguilhem ${ }^{(9)}$, un individuo afectado de heterotaxia (disposición anómala de los órganos internos) puede gozar, pues, de muy robusta salud; puede vivir mucho tiempo y, a menudo, solo después de su muerte se llegaría a advertir la presencia de una anomalía que él mismo había ignorado. Asimismo, los yoguis hindúes consiguen romper normas biológicas estadísticamente constantes. Su disciplina religiosa basada en la concentración mental les permite dominar casi completamente las funciones de la vida vegetativa: el pulso, la respiración, el electrocardiograma, la medida del metabolismo basal, etcétera. Logran colocar su cuerpo en un estado de vida retardada comparable al de los animales que invernan, siendo capaz de cambiar el pulso del ritmo cardíaco y de abolir casi total de la contracción cardíaca. Ciertamente, tales hazañas no las consideraríamos como "patologías", a pesar de alejarse de las "medias poblacionales"(9). Las excepcionales proezas de muchos atletas de alto rendimiento constituyen otro caso llamativo para ilustrar que no debe asociarse con tanta premura la "anomalía" con la "patología". Cuando estos atletas dan prueba de poder romper con marcas que parecían inalcanzables, el fisiólogo reconoce que, más que aprender del sujeto de laboratorio en situación artificial, se aprende de las selecciones profesionales en los institutos de educación física (donde los individuos concretos fijan por sí mismos los márgenes de variación tolerados por los valores biométricos). En referencia al atleta, Canguilhem no 
duda de que "son las tablas de récords, y no la fisiología, las que mejor pueden responder a la pregunta: '¿Hasta cuántos metros puede saltar el hombre?'"(9). Por estos y muchísimos indicadores más, no puede aducirse que un promedio estadístico permita decidir si el individuo que se presenta ante nosotros es "normal" o no. Al respecto, Canguilhem nos recuerda que, si hay que creerle a la tradición, Napoleón habría tenido un pulso de 40, incluso en sus días de salud:

\begin{abstract}
Si con cuarenta contracciones por minuto un organismo atiende las exigencias que se le plantean, es porque está sano y la cantidad de cuarenta pulsaciones -aunque verdaderamente aberrante con relación a la cantidad promedio de setenta pulsaciones- es normal para ese organismo. $^{(9)}$
\end{abstract}

A la luz de los muchos ejemplos que podrían mencionarse en contra de la ecuación "norma-salud" (y su opuesta, "desviaciónenfermedad"), puede afirmarse que no hay, en la naturaleza, síntomas o condiciones esperando a ser descubiertas como "enfermedades". Para que las anomalías (desviaciones de la norma estadística) Ileguen a ser tratadas como enfermedades, se requiere: 1 ) una cultura que defina un sufrimiento como morboso; 2) agentes (médicos, chamanes o sacerdotes) a los que esta sociedad reconozca como capacitados para actuar sobre ese sufrimiento; 3) individuos que acudan con tal profesional porque ellos mismos o sus allegados consideran que padecen ese tipo de sufrimiento; 4) una red de instituciones que autorice a tales actores a actuar en su nombre; 5) conocimiento verdadero, objetivo, eficiente y útil que poseen en tanto expertos ${ }^{(10)}$.

Ninguna enfermedad lo es de manera "dada": la demarcación que cada cultura traza entre la salud y la enfermedad no es fáctica, natural, sino convencional. La prueba está en que las anormalidades que permiten obtener hazañas altamente admiradas en una cultura no cuentan como enfermedades. Para poner un ejemplo sencillo, si se comprobara lo que muchas veces se ha dicho, que el cerebro de Einstein contenía un número anormal de ciertas células responsables de su genialidad, esa anormalidad no sería ciertamente considerada como una enfermedad, dado que lo que se estima son sus consecuencias y, en este caso, esas consecuencias eran muy deseables ${ }^{(11,12,13)}$. Dondequiera que se trace la diferencia entre salud y enfermedad, le subyace una distinción más profunda entre lo socialmente aceptable y lo repudiable (lo preferible y lo evitable, lo que debe promoverse y lo que debe desalentarse).

Sin embargo, "lo normal" es presentado al público no solo como "lo deseable" (y correcto) sino también como "lo naturalmente establecido" (y reflejado por el promedio). Lo que ocurre, en esta maniobra epistémica, es que se condensan en una misma palabra, "normalidad", dos sentidos que corresponden a planos distintos: al afirmarse que una condición o conducta es "normal" se alude tanto a una información descriptiva/cuantitativa (la normalidad medible, fáctica) como a un acto evaluativo (lo que se desea que se transforme en norma estadística). Este segundo aspecto valorativo es el que permanece encubierto, bajo la premisa objetivista de que la ciencia se ocupa únicamente de los hechos, sin pronunciarse por ningún valor. Quizás, uno de los efectos más sutiles y poderosos de esta confusión semántica sea que, en nombre de la experticia profesional de quien presume portar el conocimiento objetivo, se justifiquen prácticas sociales de degradación, inferiorización, estigmatización y segregación de "individuos poco ideales" (alejados de la normalidad) y exhortados a autopercibirse como "enfermos", a recibir "tratamiento" o a ingresar involuntariamente en instituciones asistenciales ${ }^{(14,15)}$.

\section{LA CONSTRUCCIÓN EPISTEMOLÓGICA DEL SOBREPESO Y LA OBESIDAD COMO ENFERMEDADES}

Es bastante común que, cuando se da a conocer un elevado incremento de los datos 
estadísticos en torno a alguna situación, se considere inmediatamente que los hechos han cambiado. Por ejemplo, el aumento del porcentaje de pacientes diagnosticados con depresión hace pensar que, actualmente, la gente se deprime más que antes; el aumento del número de casos de abuso sexual infantil induce a creer que no existió antes una época en la que haya habido tantas conductas abusivas con los niños. Se podría extender un largo compendio de ejemplos que ilustran este "realismo estadístico".

Sin embargo, la situación es bastante más compleja. No son por sí solas las estadísticas las que encienden la señal de alarma, sino su funcionamiento dentro del entramado profesional-institucional-social-político-cultural en el que adquieren pertinencia, valor y legitimidad. Ellas son parte de los "motores de descubrimiento"(2), que avanzan juntos hacia la modelación de ciertas formas de explicar un problema. Muchas de las opacidades del uso que se hace de esa información estadística quedan sencillamente ignoradas, al punto que, tras algún tiempo considerable, pareciera que el fenómeno en cuestión no puede visualizarse o explicarse de otra manera. Esta drástica confusión entre la explicación y lo explicado merece ser puesta bajo la lupa epistemológica, desde la premisa de que, como sostiene Gregory Bateson, "el mapa no es el territorio, y el nombre no es la cosa nombrada"(16). Lo que decimos de las cosas no son las cosas mismas (aunque, como ya hemos anticipado, eso que se dice, se ofrece como posible descripción y puede adquirir un sentido de realidad para quien lo adopta como creencia verdadera).

Piénsese en el siguiente caso. En los medios de comunicación de la sociedad mexicana de los últimos años, se ha instaurado la convicción de que "México es un país de gordos"; o, peor aún, que "México es (casi) el país con mayor obesidad en el mundo". Tal mensaje emana particularmente de los discursos oficiales sustentados en información estadística que avala tal afirmación; a partir del diagnóstico alarmista mostrado, se implementan campañas de salud pública orientadas a crear consciencia ante semejante situación. No obstante, dichas campañas promueven ideas que son epistemológicamente debatibles, pero que se presentan como verdades incuestionables. Por ejemplo, que "la mayoría de los mexicanos están enfermos", que "el peso corporal es un fenómeno fácil de manejar, una simple cuestión aritmética basada en un cálculo de cuánto consumes y cuánto gastas" o que "el sobrepeso y la obesidad son condiciones inevitablemente mórbidas que pueden detectarse con solo medirse".

Si bien, en México, las circunstancias que enmarcan estos discursos han sido muy particulares, hay que señalar que el estilo de pensamiento sobre la obesidad se ha ido transformando globalmente. Como bien señala Pulain $^{(17)}$, la medicina ha cambiado vertiginosamente el estatus epistémico de tal condición, la cual "ha pasado de ser considerada un simple factor de riesgo, a [considerarse] una patología crónica y más recientemente a ser una epidemia mundial"(18). En el caso específico de México, cabe recordar que, en 2010, el entonces presidente Felipe Calderón Hinojosa declaraba que México era el país con el mayor número de personas adultas con sobrepeso en el mundo, lo cual fue más tarde rectificado por la Organización para la Cooperación y el Desarrollo Económico (OCDE), al señalar que México en verdad ocupa el segundo lugar en obesidad en el mundo: según la OCDE, aproximadamente el $70 \%$ de los mexicanos padecen de sobrepeso o de obesidad ${ }^{(19)}$.

A partir de esta abrumadora presentación de "los hechos", los organismos gubernamentales implementaron varias iniciativas sanitarias tendientes a "corregir" los malos hábitos alimenticios, a fomentar la práctica de ejercicio -incluso en ámbitos laborales y escolares- y a enaltecer la importancia decisiva del "autodiagnóstico". Con este espíritu, las campañas transmitidas en los medios de comunicación masiva, en especial el televisivo, fueron incrementando el entusiasmo por instaurar, en la audiencia, una formidable confianza en estrategias de cuantificación del fenómeno; el método de medición de índice de masa corporal y el método de medición 
de circunferencia de cintura constituyen dos casos prototípicos.

Los ejemplos abundan: en 2008, el Gobierno del Distrito Federal (DF), a través de la Secretaría de Salud y los Servicios de Salud Pública del DF, lanzó la campaña "Muévete y Métete en Cintura". Como parte de la agenda de este movimiento social, los activadores físicos de la mencionada Secretaría visitaban explanadas y parques públicos, organizaban Ferias de la Salud y decenas de eventos en las 16 delegaciones de la Ciudad de México. Sobre esto, se afirmaba que "durante estos actos, nutriólogas y personal especializado realizan mediciones de índice de masa corporal (talla y peso), además de ofrecer planes nutricionales"(20). Otro caso paradigmático lo encontramos en la campaña "5 Pasos", la cual derivó del Acuerdo Nacional para la Salud Alimentaria: Estrategia contra el Sobrepeso y la Obesidad, en 2010. Luego del "Paso 1 Actívate"; del "Paso 2 Toma agua"; del "Paso 3 Come verduras y frutas"; el cuarto paso decía: "Mídete". Sucintamente, se afirmaba: "Mide tu circunferencia abdominal. Una manera sencilla de saber si tienes sobrepeso u obesidad es medirse la

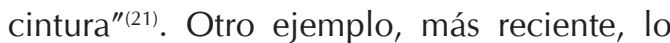
hallamos en la fotografía que acompañó la información de la noticia aparecida en enero de 2017, en la que se anunció que el Servicio de Administración Tributaria (SAT) devolverá impuestos a quien baje de peso ${ }^{(22)}$. La noticia estuvo acompañada de la imagen de un hombre midiéndose la cintura con un centímetro (su rostro no aparecía en la fotografía).

Acerca del uso de estos instrumentos de detección, autodiagnóstico y prevención del sobrepeso y la obesidad, podemos diferenciar, en principio, dos niveles de problemas. Por un lado, ellos mismos son limitados y cuestionables en tanto medios de verificación (incluso engañosos). Pero, por otro lado, alimentan una concepción hipersimplificada de los problemas asociados al peso corporal, los cuales son imaginados como si el peso dependiera exclusivamente de las cantidades de alimento ingeridas y gastadas. A continuación, se revisará escuetamente uno de los ya mencionados (y más popularizados) instrumentos de medición del peso corporal, el afamado índice de masa corporal (IMC), para pasar luego a una reflexión sobre algunas de sus incisivas implicaciones.

\section{Método de medición de índice de masa corporal}

El IMC, o índice de Quetelet es un método ideado para determinar cuál es el peso saludable de una persona, y se obtiene dividiendo su peso (expresado en kilogramos) por la altura al cuadrado (expresada en metros). Tal como afirma en Gracia Arnaiz ${ }^{(18)}$, la decisión de cuándo el exceso de peso/grasa deviene insano se ha erigido en una cuestión biomédica capital, ya que la definición de la obesidad está cimentada básicamente sobre una relación estadística epidemiológica entre IMC y patología: el umbral crítico para ser diagnosticado con obesidad $y$, por tanto, considerado enfermo, se sitúa en $30 \mathrm{~kg} / \mathrm{m}^{2}$ (mientras que el IMC óptimo se fija entre 18,5 y $25 \mathrm{~kg} / \mathrm{m}^{2}$ ).

A pesar de la aceptación más o menos generalizada de dichos parámetros, es menester señalar que el IMC no se refiere directamente a la composición corporal ni a la distribución de la grasa ${ }^{(18)}$. Existen varios modelos biomatemáticos que, atendiendo a la multidimensionalidad del tema, dividen o estructuran tal parámetro en diversas partes; por ejemplo, los modelos de cuatro componentes: grasa, músculo, hueso y tejido residual, o bien: grasa, agua corporal, componente celular y minerales en el tejido óseo; el modelo de tres componentes: grasa, masa celular activa y estructura de tejido extracelular de soporte (componente líquido y fibras proteicas); y el modelo de dos componentes: grasa y masa corporal activa ${ }^{(23)}$. Esta heterogeneidad debería proporcionar sobradas razones para que, como admite Basdevant ${ }^{(24,18)}$ el IMC sea un indicador con poco interés en el plano individual. Los contraejemplos a las estandarizaciones que la ideología del IMC impone asoman rápidamente: un musculoso fisicoculturista puede tener un IMC superior a $30 \mathrm{y}$, por supuesto, no ser obeso ${ }^{(18)}$. De forma 
similar, un atleta, especialmente de alto rendimiento, puede tener un elevado valor en el parámetro antropométrico de peso corporal, pero a partir de un alto porcentaje de músculos y de masa corporal activa en general y bajos niveles de grasa (aun cuando, al aplicar el IMC, los resultados inducirían a clasificarlo como obeso). Ni se diga lo limitante que deviene este método para evaluar atletas que compiten de acuerdo con su peso corporal, tal como acontece en el boxeo, el judo, las artes marciales mixtas o las luchas ${ }^{(23)}$. Es evidente que los criterios de obesidad emitidos por la ideología del IMC no tienen en cuenta la complejidad de los componentes, ni sus implicaciones $^{(23)}$. Sin embargo, estas tensiones no obstruyen que la ideología del IMC opere como un efectivo dispositivo de cuantificación, que además es de muy bajo costo.

El IMC (fórmula que Adolphe Quetelet introdujo en 1835) se estableció como estándar internacional por la Organización Mundial de la Salud (OMS) en 1998. México incorporó tal lineamiento, publicando en el Diario Oficial de la Federación del mismo año el proyecto de la Norma para el manejo integral de la obesidad (NOM-174-SSA1-1998), aprobada dos años más tarde. Al respecto, comenta Orellana Trinidad:

Todavía en ese momento, diversos estudios norteamericanos sugerían que el IMC no se podía aplicar a diferentes culturas. En uno de ellos, los varones italianos entre 20 y 50 años tenían índices más altos de peso que los noruegos y los norteamericanos y en otra investigación se determinó, aún con técnicas más adecuadas para medir la grasa corporal, que las poblaciones asiáticas requieren valores propios de referencia netamente por debajo de los corrientemente recomendados por la Organización Mundial de la Salud. ${ }^{(25)}$

Llevando aún más lejos la revisión epistémica del IMC, habría que agregar que no solo su generalización se vuelve problemática (considerando los mencionados riesgos de su indiscriminada aplicación masiva), sino que también su fórmula adquiere notables suspicacias. Como ya se dijo, el IMC calcula el peso del cuerpo, en kilogramos, para la altura o estatura, en metros, al cuadrado. Sin embargo, algunos críticos subrayan que, desde el punto de vista biomatemático, no es posible relacionar una medida tridimensional como el peso (que registra la resultante de los valores de altura, anchura y fondo, dimensiones anteroposteriores) con una medida lineal, como es la estatura; aunque esta sea elevada al cuadrado(23).

Ahora bien, estos atolladeros no debieran crear la ilusión de que el uso del IMC se ha visto mermado a partir de las inconformidades de sus detractores. Por el contrario, sigue funcionando como recurso privilegiado de un tratamiento uniformado que, mediante promedios y estadísticas, favorece una normalización de la corporalidad (transformando lo "anómalo" en "anormal"). Cuenta a favor de su autoconfirmación la facilidad con la cual cualquier persona puede acceder a alguna de las muchas páginas web que brindan la oportunidad de autodiagnosticarse gratuitamente con solo introducir unos datos básicos (edad, peso y altura), pudiendo determinar, así, si encaja o no encaja dentro del IMC "normal" (por ejemplo, en la página electrónica https://www.imc-peso. com). En alusión a esto, Soich y Moreno ${ }^{(26)} \mathrm{ci-}$ tan como caso destacado los sitios de acceso abierto que corresponden al que denominan "Imperio Cormillot", el cual representa a una extensa red de productos (viandas, dietas, revistas) e instancias (clínicas, clubes, programas de televisión, páginas de Internet, publicidades, spots informativos) lideradas por el afamado médico argentino Alberto Cormillot (entre ellas, la Fundación ALCO, la Clínica de Nutrición y Salud, Dietaclub, la Universidad Isalud, la franquicia Aqualife, el Instituto Argentino de Alimentos y Nutrición, la revista "Vivir mejor" y los sitios web drcormillot.com, alimentoscormillot.com, dietascormillot.com, deliverycormillot.com y kioscolight.com).

Por ejemplo, al ingresar a www.dietaclub.com, se abre la opción "Calcula tu IMC", para lo cual solicita el peso en kilogramos, la altura en centímetros y la selección 
de la contextura. A partir del IMC obtenido, se establecen cuatro categorías: peso ideal, peso posible, designación y riesgo (esto es, si se ha obtenido un IMC de 40,1, el peso ideal sería de 63,4 kilogramos, el peso posible de 74,6 kilogramos, la designación sería obesidad de tipo IV y el riesgo aparece como "muy severo"). Tras exponer estos puntos, Soich y Moreno afirman:

Podemos decir que el peso promedio establecido por el índice como "normal" tiene un equivalente estándar y objetivo que nos permite verificar el cumplimiento o no de la norma [...]. En este rango, la presunta objetividad del riesgo se basa en un cálculo de promedio aritmético como regulación fisiológica para un sujeto estándar. Con lo que podemos ver que la medición no solo es absolutamente despersonalizada a partir de un cálculo en una página de Internet, sino que además no tenemos forma de saber cuál fue ese sujeto base del cual se sacaron a priori las características del riesgo, peso y designación que constituyen un juicio de valor respecto de la normalidad de los pesos. ${ }^{(26)}$

Dicho portal exhorta a navegar por un formidable océano de ofertas; en efecto, el cálculo del IMC no se da de manera aislada, sino que se enumeran en una tabla de rápida visualización las "complicaciones" y los trastornos del "ánimo" asociados a cada nivel (a mayor patología del peso, mayor correlación con el enojo, la frustración, la depresión, los miedos, la agresividad, la hostilidad, la ideación suicida, entre otros). Es oportuno aclarar que el estilo "pedagógico" que predomina en tal sitio omite el lenguaje hipotético: se presentan "los hechos" como si se trataran de certezas médicas, y no de perspectivas controversiales y en permanente revisión. Cuando se subraya que la mayoría de las personas que cumplen con el IMC igual o superior a $30 \mathrm{~kg} /$ $\mathrm{m}^{2}$ presentan un futuro deterioro, no se tiende a hablar de tal anticipación como una posibilidad, sino como una evidencia. Asimismo, el espacio web alude también a las infaltables "opciones terapéuticas" (bajo el nombre de "Conoce nuestros planes"). Entre otras cuestiones, hay que resaltar que, en la definición de la obesidad oficializada por la "industria Cormillot", se destaca que esta es "una enfermedad en la que la gran mayoría de las personas que toman la decisión firme y sostenida de recuperarse, pueden hacerlo"; al ponerlo en esos términos, la Clínica de Nutrición y Salud del Dr. Cormillot:

...instituye valoraciones claramente orien-
tadas a subrayar la responsabilidad indi-
vidual y la elección racional respecto del
cuidado de sí -entendido, claro está, úni-
camente como pérdida de peso y ajuste
de la corporalidad "anómala" al patrón
estadístico. ${ }^{(26)}$

\section{La construcción caracterológica del sobrepeso y la obesidad}

Las pretensiones epistémicas del abordaje del sobrepeso y la obesidad como "patologías" no vienen solas. Por el contrario, están influidas por poderosas inferencias acerca de cómo el "gordo" o el "obeso" piensa, siente y actúa. Tales estereotipificaciones justifican el aspecto médico del problema (el obeso está enfermo) pero también les concede injerencia a otras profesiones asociadas, tales como la psiquiatría, la psicología, la psicoterapia y la psicofarmacología. Sobre la predominancia de ciertas representaciones caracterológicas de la gordura en la cultura accidental, Fischler ${ }^{(27)}$ identifica dos patrones icónicos: el gordogoloso, aceptado socialmente por simpatía, y el gordo-glotón, rechazado por ser egoísta y ocioso. Fischler considera que este último es el que se ha sobreimpuesto, bajo el imaginario de que las personas obesas comen desenfrenadamente, transgrediendo las normas de la compartición. La gordura es considerada física y moralmente insana, obscena, propia de perezosos y mezquinos (cualidades asociadas con la autoindulgencia). En contraste, la delgadez no solo es presentada como atractiva, sino que se asocia con la mesura, el esfuerzo y la metodicidad (virtudes emparentadas con el 
autocontrol). Como bien lo establece Gracia Arnaiz ${ }^{(18)}$, el rechazo sistemático de las grasas y el temor a engordar ha alcanzado, en las sociedades industrializadas, una intensidad inusual, deviniendo, incluso, una suerte de lipofobismo. Su consolidación como "problema sanitario" no solo coincide, en el siglo $X X$, con la consecutiva transformación del estatus epistemológico otorgado por la medicina a la gordura como enfermedad, sino también con la concurrencia de otros aspectos sociohistóricos no menos importantes:

Hoy los juicios negativos sobre la gordura son mayoritariamente compartidos. En esta negativización han jugado un papel relevante: la moral del Occidente cristiano reclamando prudencia y mesura en la comida y menospreciando la glotonería, la evolución del conocimiento científico demostrando la estrecha relación entre dieta y salud, y los cambios habidos en las representaciones del cuerpo, que han convertido la delgadez en signo de salud y distinción social y la obesidad en todo lo contrario. ${ }^{(18)}$

El marco actual dentro del cual se observa el problema del sobrepeso y la obesidad se ha configurado bajo el ala de la normalización dietética y corporal, cuyo paso ha sido empujado por la interpretación moral que la ciencia ha hecho de las denominadas "sociedades de la abundancia". La autora advierte que, en esta época de una exuberancia alimentaria sin precedentes, la gordura no es más que una especie de "falla" que acompaña el proceso civilizatorio. Transgredir la norma por exceso implica dar pruebas de indisciplinamiento. Poco importa que la medicina misma reconozca que la excesiva acumulación de grasas se relaciona con numerosas causas funcionales de tipo metabólico, genético, medicamentoso u hormonal. Desde la convicción de que el gordo es un autopermisivo "gran comedor" que consume el máximo y gasta el mínimo, será acusado de ser una especie de delincuente nutricional(24). Tenderá a ser culpado de sufrir la enfermedad, aun cuando las enfermedades son, por definición, involuntarias, padecidas. Por si fuera poco, la abrumadora estigmatización de tales sujetos puede ir frecuentemente acompañada de una historia familiar en la que, de antemano, se asume que no le marcaron límites a su voracidad ante la comida (con lo cual adviene una re-estigmatización: hijo gordo: hijo de padres complacientes e indulgentes, la "dejadez" como lastre y huella familiar). Ante tal asignación de un rol que, en el mejor de los casos, se lo atribuye a la ignorancia, la intervención del profesional reviste la mayor importancia: debe inculcar el estilo de vida saludable, debe enseñar a comer, debe extirpar los malos hábitos, debe impulsar a la prevención y advertirles a los pacientes de los riesgos del sobrepeso y la obesidad. En tal esfuerzo, debe quedar demostrado que la ciencia es la mejor justificación de la autonormalización, y que el afrontamiento económico de los tratamientos de la industria dietológica y bariátrica están más que validados por la gravedad "epidémica" de la obesidad a nivel mundial.

Este tipo de explicación permite, además, hacer recaer el fracaso terapéutico sobre el paciente (exonerando, así, a quien ha indicado el tratamiento), ya que la misma desidia personal y familiar que ha promovido la obesidad, podría ahora favorecer el abandono rápido de las medidas correctivas aplicadas por el médico. Asimismo, el intento fallido de autonormalización podría ir acompañado, en este caso, de una auto-re-estigmatización (el sujeto se rechaza a sí mismo por ser gordo, pero también por no poder lidiar con el problema ni siquiera cuando los profesionales se esmeran para "ayudarlo"). La etiquetación, que deriva de los intercambios interaccionales con el entorno, puede moldear dramáticamente su autopercepción e inducir a diferentes formas de autodesvalorización y autosegregación.

\section{LA OTRA EPIDEMIA: LAS GENERALIZACIONES}

Como advierte Hacking ${ }^{(28)}$, hay sobre la obesidad dos niveles epidémicos en los 
discursos dominantes: por un lado, el de la obesidad misma, establecida según escalofriantes datos estadísticos; por el otro lado, el del discurso sobre la obesidad, en torno al cual giran las múltiples narrativas moldeadas por las voces de la comunidad biomédica (narrativas que, según Hacking, se sintetizan en el título del artículo de Le Fígaro, que reza así: "La obesidad, mal del siglo"). Ambos niveles (el estadístico-poblacional y el calificativo-catastrofista) se retroalimentan, y ello queda ilustrado en el subtítulo del artículo de Le Fígaro: "Un francés de cada diez sufre de grave exceso de peso"(28). No hay país que no tenga sus propias estadísticas sobre la tasa de mortalidad asociada a la obesidad: un infierno para cada observatorio nacional.

Ahora bien, desde el punto de vista epistemológico, podríamos hablar de un tercer nivel epidémico: el de las generalizaciones, las cuales abundan en las formas de presentar, explicar y difundir dicho problema. Cuando se generaliza una idea, lo sutilmente diferente, lo novedoso, lo intrigante, lo irresuelto, lo ambiguo, lo confuso o lo inesperado de cada situación particular es borrado para hacerlo encajar dentro de un marco de explicación que no admite fácilmente las excepciones o los matices. Dicha monopolización conceptual, que actualmente es detentada por la ciencia, pretende cartografiar un mundo en el que la inteligibilidad de los fenómenos solo parece asequible si se establecen leyes naturales, expresadas como universalizaciones o como generalizaciones.

Pero, precisamente, aquí se enquista la trampa epistemológica: en las campañas de "concientización del problema" que mediatizan las políticas de salud pública, no se vislumbran esfuerzos por evitar la creencia lineal (y errónea) de que todos los cuerpos acumulan grasas del mismo modo si se ingiere la misma cantidad de alimentos ${ }^{(29)}$; tampoco se desestima la visión del gordo como un gran comedor pasivo, ni se promueve una educación nutricional que genere oportunidades de pensar sobre la cuestión nutricional como un fenómeno complejo y multidimensional; no se cuestiona tampoco el dogma simplista según el cual, quien padece objetivamente sobrepeso u obesidad, tiene que sentirse necesariamente enfermo; ni se incentiva una visión enriquecida acerca de cómo entender la intrincada noción de "estilo de vida saludable". Por el contrario, se insiste en la naturalización de la idea de que los problemas alimentarios $y$, en particular, aquellos relacionados con el peso, tienen su origen en la cantidad de comida ingerida, como si los efectos de comer en el cuerpo fueran una simple operación aritmética: se come poco o demasiado y engordamos o adelgazamos según las calorías consumidas(18). El cálculo calórico supone una invisibilizada transformación de nociones cualitativas (como cuando se dice de alguien que es "rellenito", "robusto", "corpulento", "flaco", "delgado") en nociones con precisión cuantitativa (normopeso, sobrepeso, obesidad de tipo I, II, III), términos que pueden determinarse mediante el uso de una balanza, un metro y una norma, como la del IMC.

La estandarización del diagnóstico encapsula las opacidades epistemológicas que nos harían desconfiar de esos discursos que se presentan como portadores de verdades consagradas y definitivas (cuando, en realidad, lo que codifican son ideas consensuadas). Como dice Gracia Arnaiz:

Ni todos los gordos están enfermos ni
todos comemos mal. Es más, ni siquiera
todos los que comemos mal o no hace-
mos ejercicio a diario acabaremos
siendo gordos. Ciertamente, no todos
estamos igualmente amenazados por la
obesidad, aunque el discurso actual y las
medidas de prevención que lo acompa-
ñan hacen creer lo contrario. ${ }^{(18)}$

Por extraño que parezca, hay casos en los cuales la condición de sobrepeso no significa necesariamente una aflicción, incomodidad, impedimento, incompetencia o morbilidad, por lo cual las generalizaciones emanadas de esta representación mediática del fenómeno solo hablan de un sujeto abstracto, desarraigado, sin biografía. Las estrategias discursivas que así lo consideran no hacen sino reafirmar las tácticas intrínsecas 
a la biopolítica que tiene en su eje a un Estado técnico-científico que interviene los cuerpos para socializarlos a través de la red de instituciones que administran la vida individual, familiar y ciudadana. Ni las narrativas sobre la alimentación ni las narrativas sobre el deporte podrían sustraerse, hoy en día, a los imperativos del discurso de la salud ${ }^{(30)}$. El cuerpo ha quedado atrapado en los tentáculos de los dispositivos de poder que obligan a regimentarse crónicamente en pro del nuevo cuidado de sí. Remitiéndose a los renovados ascetismos por el cuerpo perfecto, Laura Contrera afirma:

En el cruce entre fitness e industria de la dieta vemos cómo la salud -y la apariencia saludable- son deseo individual y lucro empresarial a la vez. La gordura, hoy definida como una epidemia de alcance mundial con contornos bien especificados, es un punto nodal de este cruce, ya que su presencia -o ausencia- habilita el pase al equipo de los cuerpos patológicos/indeseables o normales/deseables. Pero la gordura no es como cualquier otra enfermedad que pueda contraerse en una sociedad preocupada por la salud: se la asocia al consumo excesivo de alimentos tanto como al deficiente y también al modo de vida nocivo de seres sin voluntad. El dispositivo de corporalidad produce a la gordura como una tara del cuerpo, haciéndola un índice de falta de autocontrol (y demás valores del mercado como la rentabilidad, la eficiencia, competitividad, alto desempeño, etc.), por eso se la asocia al fracaso social. ${ }^{(31)}$ (Cursivas añadidas)

Observando el fenómeno desde la óptica del mundo neoliberal, que enmarca a las políticas sanitarias en muchos países, Costa ${ }^{(32)}$, citado por Contreras ${ }^{(33)}$, subraya que:

...esta batería de tecnologías del yo -cosméticas, quirúrgicas, gimnásticas, dietéticas, farmacológicas- permite una mayor competitividad en los mercados de trabajo, de prestigio, de status social o de deseo, a la vez que exacerba que las diferencias socioeconómicas se vuelvan diferencias físicas, estéticas.

"Mejorar" es "encajar", es hacer coincidir el lema biopolítico médico-institucional: "La gordura es enfermiza" ("Fat is unhealthy") con el lema subjetivizado, e introyectado "La gordura es repugnante" ("Fat is bad"). Independientemente de que uno tenga o no sobrepeso u obesidad, se promueve que uno viva (y desee vivir) en permanente estado de alerta o autovigilancia:

...gordo/a no es un mero adjetivo calificativo que describe una cualidad sustantiva del cuerpo, sino que es un insulto, así como también acusación (dejadez, falta de cuidado), diagnóstico (enfermedad) y sentencia (muerte física o social). ${ }^{(33)}$

El capitalismo funciona simultáneamente como "una fabulosa máquina de producción de exceso y falta que permite que el fantasma del hambre y el fantasma de la gordura horroricen a los sujetos contemporáneos", según Sibilia ${ }^{(34)}$, citado por Contreras ${ }^{(33)}$. En otras palabras: el sujeto actual es un sujeto disponible: disponible para el atracón, y también disponible para ser purgado de esa sobrealimentación. Y para cada uno de esos momentos hay una abrumadora infraestructura médica-industrial-empresarial-dietológica.

La frecuente miopía histórico-antropológica bajo la cual pensamos el peso corporal nos priva de un abordaje del problema que nos permita tener una perspectiva comparativa sobre la enorme variabilidad de sentidos que cada sociedad, grupo o comunidad ha construido históricamente en torno a las nociones de nutrición, salud, cuerpo y bienestar. Fuera de un enfoque contextualizado, las definiciones actuales parecen correctas simplemente por ser las vigentes. Un recorrido panorámico por la historia de otras culturas y otras épocas nos dejaría constatar que el imperante lipofobismo contemporáneo no ha sido una constante universal; ante ello, bien podríamos preguntarnos en qué momento y 
bajo qué combinación de circunstancias la alta valoración de las grasas y de los alimentos energéticos (previamente apreciados, en cantidad moderada, como barrera de resistencia ante casos de enfermedad) se convirtió en una declarada guerra contra la grasa y la acumulación calórica. Pensar cuantitativamente acerca de la alimentación ya no tiene que ver con la antigua preocupación de la insuficiencia de comida (esto es, con el temor de que no alcance, tal como ocurría en las sociedades de la escasez) sino con la regulación y restricción de la ingesta individual ante la prodigalidad de alimentos (típico de las sociedades de la abundancia).

Es seguro que, dentro de la ecología de creencias, valores y prácticas que favorecieron este viraje, hay una plétora de factores diversos y concatenados. La injerencia biomédica y la definición del problema como un asunto de interés político-sanitario es una de las tantas aristas del tema, como lo es también el peso relativo que las jugosas compañías de seguro médico vislumbraron como oportunidad. El antropólogo social Jesús Contreras señala que, ya desde mediados del siglo XIX, las aseguradoras usaban el peso corporal como un indicador de riesgo ${ }^{(33)}$. Considera que la Dublin's estandar table of heigts and weigths, de 1908, fue decisiva en el establecimiento de los primeros promedios de peso ideal. Tampoco pueden eludirse otros aspectos que ya han sido hondamente estudiados, particularmente desde la trinchera del denominado activismo de la grasa; por ejemplo, el concomitante cambio en los estereotipos de belleza, así como la invasión del "imperio del régimen" en los medios de comunicación de masas y la edición, el marketing y la publicidad, la medicina y las medicinas "paralelas", "suaves" o "alternativas"(33), la sedentarización de la fuerza de trabajo $y$, en especial, la incidencia de transformaciones socioculturales que tienen una relevancia tan fuerte como los clásicamente ponderados aspectos biológicos sobre las costumbres nutricionales, las cuales impregnan prácticas culturales que no se sostienen pura y exclusivamente en razón de la salud, sino por otros motivos igualmente importantes. La cantidad de alimentos que los humanos pueden ingerir no depende únicamente de cambiantes necesidades fisiológicas. El aumento de la capacidad adquisitiva, la creciente atención hospitalaria y la ostentación festiva/ceremonial, pueden incitar a un mayor consumo y a una demanda creciente de alimentos socialmente prestigio$\operatorname{sos}^{(33)}$. A través de la comida, las personas no buscan halagar únicamente a los nutricionistas, ni deciden acatar ciegamente las indicaciones de los profesionales de la salud, aun cuando confían en su conocimiento acerca de los efectos nocivos de ciertas formas de alimentarse. Muchas mujeres adoptan regímenes o dietas a sabiendas de que no son las más adecuadas, o persisten en fumar por temor a engordar ${ }^{(33)}$. Es decir, no hay una relación lineal y directa entre el conocimiento dietológico y la toma individual de decisiones. Por ende, el abordaje centrado básicamente sobre lo que esos individuos hacen o no hacen, omite profundamente el carácter multifacético que la cuestión reviste.

\section{CONCLUSIÓN}

Comer es un acto biológico, pero "comer de cierta forma" es un ejercicio político, que involucra preferencias, valoraciones y modos de relación con uno mismo y con los demás. Lo mismo puede decirse de la actividad física. Ahora bien, los esfuerzos por disciplinar, normalizar y uniformar las formas en que nos alimentamos, nos activamos y vivimos no clausuran la repudiada diversidad corporal. Ciertamente, el reduccionismo discursivo que irradia de las mencionadas campañas de sensibilización, detección y prevención de las enfermedades, presupone que los ciudadanos reaccionarán masivamente con "racionalidad dietética"; pero, como dice Samantha Murray ${ }^{(35)}$, citado por Contrera(31) "no hay una experiencia única de la gordura que produzca una identidad gorda homogénea: las maneras de vivir un cuerpo gordo son siempre múltiples, contradictorias y eminentemente ambiguas". 
Retomando las reflexiones de Canguilhem $^{(9)}$ acerca de la carga valorativa de los juicios que hacemos sobre la experiencia de "sentirse enfermo", podemos enfatizar que ni el índice de masa corporal, ni la medida de circunferencia de cintura, ni el peso, ni la altura ni la complexión de una persona nos dice algo sobre su bienestar o su salud. Catapultar los cuerpos gordos como "enfermos" no solo deriva de un prejuicio moral, sino también de un sesgo epistemológico. En realidad, ambos se retroalimentan: lo que es moralmente malo, lo es porque hay evidencias científicas de ello. Esa poderosísima mancuerna ideológica -ética/ epistemológica- opera en muchos programas de salud colectiva creados explícitamente para dar una solución sanitaria a los problemas sociales: recurrentemente se basan en flagrantes generalizaciones, aderezadas de cifras estadísticas que carecen de contexto, de tradición y de raíces idiosincrásicas. Ello constituye un paso más hacia la construcción de la subjetividad como corporalidad cuantificada; los sujetos quedan expuestos a la construcción de su identidad en términos de una especie de homo caloricus, domesticado para autodespreciarse en su singularidad no-ideal.

Los enfoques acerca de los hábitos de la nutrición, del cuidado del cuerpo y de la búsqueda de salud son, por supuesto, innumerables; por lo cual, la perspectiva biomédica dista mucho de agotar el tema. No obstante, su retórica ha colonizado los medios de comunicación instaurando el fenómeno del sobrepeso y la obesidad como cuestiones ante las cuales la ciencia debe mostrar una función epistémica (investigar los procesos alimentarios desde un plano biológico-empirista), una función instructiva (enseñarle a los pacientes a comer y a hacer actividad física) y una función burocrática-administrativa (participar de forma comprometida en los diseños de políticas de salud pública de carácter terapéutico y preventivo). La sensación de que el problema de la obesidad es una amenaza mundial empuja a la búsqueda de soluciones exitosas y urgentes. Obviamente, ante un escenario que aparenta ser tan apremiante, la aletargada reflexión filosófica no es mirada como un campo fértil para hallar respuestas con valor pragmático probado y visible. Por ello, se hacen esmerados intentos de maximizar el potencial científico de las disciplinas que mejor se prestan al análisis estadístico, al diagnóstico estandarizado, y a la compulsión por las clasificaciones presuntivamente objetivas. Dado que existe un viralizado pavor hacia las anunciadas consecuencias médicas y sociales de la obesidad, pareciera que el pensamiento crítico-epistemológico sobre las plurales formas de vivir la corporalidad fuera un lujo anacrónico y nostálgico.

\section{REFERENCIAS BIBLIOGRÁFICAS}

1. Efran JS, Lukens MD, Lukens RJ. Lenguaje, estructura y cambio: la estructuración del sentido en psicoterapia. Barcelona: Gedisa; 1990.

2. Hacking I. Façonner les gens. En: Hacking I. Leçon inaugurale: chaire de philosophie et histoire des concepts scientifiques [Internet]. Paris: Collège de France; 2001-2002 [citado 10 ago de 2017]. Disponible en: https://tinyurl.com/y7wkeo8q.
3. Hacking I. Biopower and the avalanche of printed numbers. Humanities in Society. 1982;5(3-4):279295.

4. Hacking I. World-making by kind-making: child abuse for example. In: Douglas M, Hull D L, (eds.). How classification works: Nelson Goodman among the social sciences. Edinburgo: Edinburgo University Press; 1992. p. 180-237.

5. Hacking I. The looping effects of human kinds. En: Sperber D, Premack D, Premack AJ, (eds.). 
Causal cognition: a multidisciplinary debate. New York: Oxford University Press; 1994. p. 351-383.

6. Hacking I. Rewriting the soul. Princeton: Princeton University Press; 1995.

7. Hacking I. Taking bad arguments seriously. London Review of Books. 1997;19(16):14-16.

8. Hacking I. Social construction of what? Harvard: Harvard University Press; 1999.

9. Canguilhem G. Lo normal y lo patológico. Buenos Aires: Siglo XXI Editores; 1983.

10. Fernández Liria A. De la psicopatología crítica a la crítica de la psicopatología. Revista de la Asociación Española de Neuropsiquiatría. 2001;21(80):57-60.

11. Scheff TJ. Labelling madness. Englewood Cliffs: Prentice Hall; 1975.

12. Reznek L. The nature of disease. New York: Routledge \& Kegan Paul; 1987.

13. Reznek L. The philosophical defence of psychiatry. New York: Routledge; 1991.

14. Goffman I. Asylums: essays on the social situation of mental patients and other immates. Garden City: Doubleday; 1961.

15. Goffman I. Estigma: la identidad deteriorada. Buenos Aires: Amorrortu; 2003.

16. Bateson G. Espíritu y naturaleza. Buenos Aires: Amorrortu; 1979.

17. Poulain JP. Sociologie de I'obesité. Paris: Presses Universitaires de Paris; 2009.

18. Gracia-Arnaiz M. La medicalización de la obesidad: concepciones y experiencias sobre la gordura en jóvenes con "exceso" de peso. Zainak. 2011;34:225-241.

19. Instituto Nacional de Salud Pública, Secretaría de Salud del Distrito Federal. Encuesta nacional de salud y nutrición 2012: resultados nacionales [Internet]. Cuernavaca: Instituto Nacional de Salud Pública; 2012 [citado 18 ago 2017]. Disponible en: https://tinyurl.com/ychramay.

20. Secretaría de Salud del Distrito Federal, Ciudad de México. Campaña "muévete y métete en cintura" [Internet]. México DF: Secretaría de Salud del Distrito Federal; 2008 [citado 20 ago 2017]. Disponible en: https://tinyurl.com/y7kmt8fu.

21. Secretaría de Salud del Distrito Federal. 5 Pasos
[Internet]. México DF: Secretaría de Salud del Distrito Federal; 2011 [citado 22 ago 2017]. Disponible en: http://www.5pasos.mx.

22. Devolverá SAT impuestos a quien baje de peso [Internet]. El Universal Compañía Periodística Nacional SA; 2017 [citado 20 ago 2017]. Disponible en: https://tinyurl.com/yahtrjvu.

23. Alonso López RF. Índice de masa corporal (IMC): aciertos y desaciertos [Internet]. EFDeportes. com Revista Digital; 2011 [citado 13 ago 2017]. Disponible en: https://tinyurl.com/y9vqksf2.

24. Basdevant A. But et abuses de la définition medical contemporaine. En: Csergo J, (coord.). Trop gros?: I'obesité et ses representations. París: Éditons Autrement; 2009. p. 112-122.

25. Orellana Trinidad L. Obesidad y consumo [Internet]. El Siglo de Torreón; 2010 [citado 11 ago 2017]. Disponible en: https://tinyurl.com/ yd78oe8p.

26. Soich M, Moreno ML. Tras las huellas del "Hombre de Cormillot": una aplicación argentina de la perspectiva de los Fat Studies para el análisis de un dispositivo de normalización corporal. En: García E, Fortunato A. Actas de las I Jornadas Internacionales Filosofías del Cuerpo / Cuerpos de la Filosofía. Buenos Aires: Facultad de Filosofía y Letras Universidad de Buenos Aires; 2014. p. 180188.

27. Fischler C. El (H)omnívoro. Barcelona: Anagrama; 1995.

28. Hacking I. Leçon 3: normalisation et obesité [Internet]. Paris: Collège de France; 2005 [citado 11 ago 2017]. Disponible en: https://tinyurl.com/ ycxytutu.

29. Alemany M. Mecanismos de control del peso corporal. Revista de la Real Academia de Medicina de Cataluña. 2003;18(2):44-49.

30. Alú M. El cuerpo como objeto de las políticas públicas: de qué hablamos cuando hablamos del cuerpo político. En: García E, Fortunato A. Actas de las I Jornadas Internacionales Filosofías del Cuerpo / Cuerpos de la Filosofía. Buenos Aires: Facultad de Filosofía y Letras Universidad de Buenos Aires; 2014. p. 12-17.

31. Contrera L. El cuidado de los cuerpos impropios: gordura, revueltas y dietas en las sociedades de control/seguridad. En: García E, Fortunato A. Actas de las I Jornadas Internacionales Filosofías del Cuerpo / Cuerpos de la Filosofía. Buenos Aires: Facultad de Filosofía y Letras Universidad de Buenos Aires; 2014. p. 58-62. 
32. Costa F. El dispositivo fitness en la modernidad biológica; democracia estética, just-in-tie, crímenes de fealdad y contagio [Internet]. Jornadas de Cuerpo y Cultura de la UNLP, La Plata, 2008 [citado 22 ago 2017]. Disponible en: https:// tinyurl.com/yawlh4hu.

33. Contreras Hernández J. La obesidad: una perspectiva sociocultural. Formación Continuada en Nutrición y Obesidad. 2002;5(6):275-286.
34. Sibilia P. Pureza y sacrificio: nuevos ascetismos por el "cuerpo perfecto". Artefacto: Pensamientos Sobre la Técnica. 2007;(6):38-44.

35. Murray S. (Un/be) Coming out?: rethinking fat politics. Social Semiotics. 2005;15(2):153-163.

\section{FORMA DE CITAR}

Christiansen ML. "Homo Caloricus": La construcción epistemológica del estigma lipofóbico en los discursos mediáticos de salud pública. Salud Colectiva. 2018;14(3):623-637. doi: 10.18294/sc.2018.1602.

Recibido: 6 de septiembre de 2017 | Versión final: 26 de diciembre de 2017 | Aprobado: 18 de abril de 2018

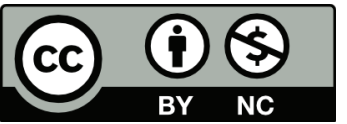

Este obra está bajo una licencia de Creative Commons Reconocimiento-NoComercial 4.0 Internacional. Reconocimiento - Permite copiar, distribuir y comunicar públicamente la obra. A cambio, se debe reconocer y citar al autor original. No Comercial — Esta obra no puede ser utilizada con finalidades comerciales, a menos que se obtenga el permiso. 